\title{
Pullout Strength Predictor: A Machine Learning Approach
}

\author{
Ravi Khatri ${ }^{1,2}$, Vicky Varghese ${ }^{1}$, Sunil Sharma ${ }^{3}$, Gurunathan Saravana Kumar $^{2}$, Harvinder Singh Chhabra ${ }^{4}$ \\ ${ }^{1}$ Biomechanics Lab, Indian Spinal Injuries Centre, New Delhi, India \\ ${ }^{2}$ Department of Engineering Design, IIT Madras, Chennai, India \\ ${ }^{3}$ Indian Spinal Injuries Centre, New Delhi, India \\ ${ }^{4}$ Department of Spine Surgery, Indian Spinal Injuries Centre, New Delhi, India
}

\begin{abstract}
Study Design: A biomechanical study.
Purpose: To develop a predictive model for pullout strength.

Overview of Literature: Spine fusion surgeries are performed to correct joint deformities by restricting motion between two or more unstable vertebrae. The pedicle screw provides a corrective force to the unstable spinal segment and arrests motions at the unit that are being fused. To determine the hold of a screw, surgeons depend on a subjective perioperative feeling of insertion torque. The objective of the paper was to develop a machine learning based model using density of foam, insertion angle, insertion depth, and reinsertion to predict the pullout strength of pedicle screw.

Methods: To predict the pullout strength of pedicle screw, an experimental dataset of 48 data points was used as training data to construct a model based on different machine learning algorithms. A total of five algorithms were tested in the Weka environment and the performance was evaluated based on correlation coefficient and error matrix. A sensitive study of various parameters for obtaining the best combination of parameters for predicting the pullout strength was also preformed using the L9 orthogonal array of Taguchi Design of Experiments.

Results: Random forest performed the best with a correlation coefficient of 0.96 , relative absolute error of 0.28 , and root relative squared error of 0.29 . The difference between the experimental and predicted value for the six test cases was not significant $(p>0.05)$. Conclusions: This model can be used clinically for understanding the failure of pedicle screw pullout and pre-surgical planning for spine surgeon.
\end{abstract}

Keywords: Pedicle screw; Machine learning; Osteoporosis; Decision support; Pullout; Polyurethane foam; Implant; Spine; Spine fusion

\section{Introduction}

Lower back pain is the second most common ailment that affects $80 \%$ of people in their lifetime. Commonly observed causes of lower back pain are spinal instability, degenerative disc disease, infection, and other idiopathic reasons [1]. Pain due to mechanical spine instability is treated by spine fusion surgery, which involves either an instrumented or non-instrumented technique. These surgeries prevent motion at painful joints and correct defor-

Received Sep 21, 2018; Revised Feb 5, 2019; Accepted Mar 10, 2019

Corresponding author: Harvinder Singh Chhabra

Spine Services, Indian Spinal Injuries Centre, New Delhi, 110070, India

Tel: +91-11-42255243, Fax: +91-11-2689 8810, E-mail: drhschhabra@isiconline.org 
mities between unstable vertebrae. For an instrumented spine fusion surgery, the unstable motion segments are rigidly stabilized using pedicle screw and rod fixation. This aids in providing necessary load sharing construct for bone fusion and decompression of nerve roots which relieves back pain [2]. Despite considerable advances in pedicle screw design, failures such as screw pullout and breakages continue to be reported. One of the major causes of pedicle screw failure is the weak screw and bone interface, attributed to osteoporosis at the vertebral bodies that reduces strength at the screw-bone interface resulting in construct failure. Failure of the pedicle screws may lead to treatment setbacks, painful pseudo arthrosis, and prolonged rehabilitation [3].

Pullout strength is an important index to objectively understand the stability of fusion construct. Factors such as bone mineral density (BMD), angle of insertion, screw length, screw thread parameters, etc. influence the pullout phenomenon [4-8], which have been examined through experiments on foam and cadaver models [9-11]. Clinically, the pullout strength correlates to insertion torque which is very subjective and depends on the surgeon's experience $[12,13]$. Since insertion torque is observed peri-operatively, there is a need to develop a technique to measure pullout strength preoperatively, which can aid in pre-surgical planning.

Machine learning is an emergent and interdisciplinary field within the mathematical and computational sciences that can be applied to a range of activities such as finance, traffic control, email spam filtering, etc. The premise of machine learning is to enable a computer to learn and predict accurate outcomes without being explicitly programmed [14]. It does so through acquisition of knowledge via data mining, discovery of new facts and theories by observation and experimentation, and then making predictions in an acceptable range using statistical analysis. There has been growing demands for application of machine learning in the healthcare sector for clinical predictions. Particularly in spine fusion surgeries, machine learning algorithms can play a pivotal role during presurgical planning to increase the accuracy of diagnoses, and also aid the surgeon in avoiding human errors [15]. The objective of the current study was to develop a machine learning based model based on previously published experimental data that can be applied for prediction of pedicle screw pullout strength.

\section{Materials and Methods}

\section{Machine learning algorithms}

Since the objective was to demonstrate the application of machine learning algorithms for prediction of pullout strength, the detailed description of algorithms used were not discussed in the current paper, but a brief description of various algorithms used in the study is provided as supplementary data for the benefit of readers who are new to this field.

\section{Pullout strength analysis}

Pullout strength test was performed on rigid polyurethane foam (Fig. 1) used as a surrogate representing normal to osteoporotic bone. Polyaxial pedicle screws (GESCO Health Care Pvt. Ltd., Chennai, India) measuring $6 \times 40$ $\mathrm{mm}$ and $7 \times 40 \mathrm{~mm}$ and made of medical grade titanium alloy were used. Tensile load of $5 \mathrm{~mm} / \mathrm{min}$ was applied to the test specimen until the maximum load was reached in load versus displacement graph as per ASTM 543-07 (https://www.astm.org/Standards/F543.htm) [16].

\section{Machine learning model}

Training data for the model was incorporated into an experimental table that was built using L32 orthogonal array consisting of four factors-density, insertion depth, insertion angle, and reinsertion (Table 1). Input dataset consisted of 48 data points as per the Taguchi L32 Design of Experiments.

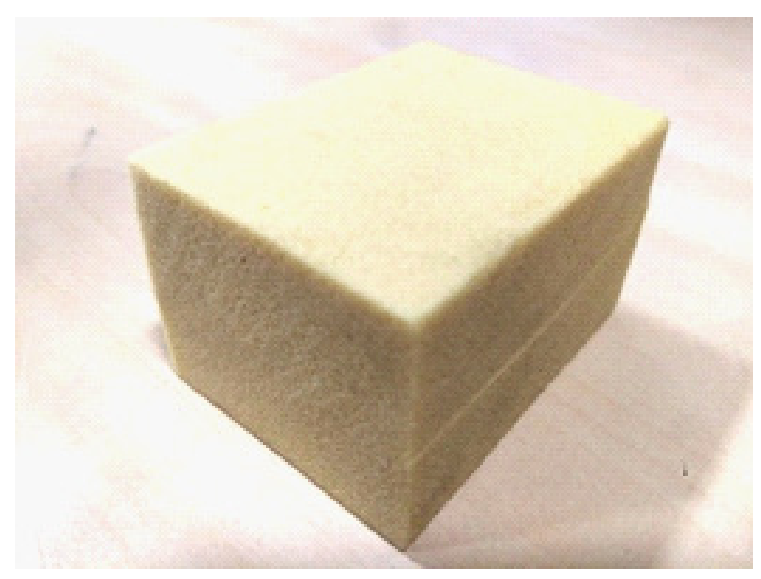

Fig. 1. Rigid polyurethane foam with $300 \mathrm{~kg} / \mathrm{m}^{3}$ density. 


\section{Machine learning algorithm evaluation}

Machine learning algorithms were evaluated to predict the pullout strength of a pedicle screw using Waikato Environment for Knowledge Analysis (Weka; The University of Waikato, Hamilton, New Zealand), a popular suite of machine learning algorithms. Four different classes of Weka classification algorithms namely, lazy, meta, and trees were used, and the performance was evaluated in terms of correlation coefficient and error matrix. The error matrix consisted of mean absolute error, root mean squared error, relative absolute error, and root relative squared error $[17,18]$. Pullout strength experimental data from test conducted by author was used for evaluation of machine learning algorithm of Varghese et al. [19].

Table 1. Selected variables for pullout strength studies

\begin{tabular}{llrrrr} 
Serial no. & \multicolumn{1}{c}{ Factors } & \multicolumn{4}{c}{ Levels } \\
\hline 1 & Density $\left(\mathrm{kg} / \mathrm{m}^{3}, \rho\right)$ & 80 & 160 & 240 & 300 \\
2 & Insertion depth $(\%, l)$ & 70 & 80 & 90 & 100 \\
\hline 3 & Insertion angle $\left({ }^{\circ}, \theta\right)$ & 0 & 10 & 20 & 30 \\
4 & Reinsertion & 0 & 1 & Nil & Nil \\
\hline
\end{tabular}

\section{Sensitivity analysis}

A sensitivity analysis was performed by varying the parameters of the best performing algorithm as per L9 array of Taguchi Design of Experiments. This analysis enabled identification of the optimum combination for predicting pullout strength. This combination was used to evaluate the prediction of the outcomes of six test cases that were not used for building the model. Two sample Student $t$ test with equal variance at $p<0.05$ level of significance was used for data analysis.

\section{Results}

The performance of machine learning algorithms used to build the model for pullout strength prediction is presented in Table 2. The correlation coefficient was the highest

Table 3. Levels at which parameters were evaluated using L9 orthogonal array

\begin{tabular}{lrrr} 
Random forest parameters & \multicolumn{3}{c}{ Levels } \\
Seed & 2 & 4 & 6 \\
No. of features & 0 & 2 & 4 \\
No. of Iterations & 10 & 50 & 100 \\
\hline
\end{tabular}

Table 2. Performance of different algorithms

\begin{tabular}{llllllll} 
Serial no. & Class & Algorithm & CC & MAE & RMSE & RAE (\%) & RRSE (\%) \\
\hline 1 & Lazy & Kstar & 0.89 & 136.75 & 164.99 & 44.69 & 46.16 \\
2 & Lazy & LWL & 0.80 & 172.25 & 204.61 & 56.30 & 57.24 \\
\hline 3 & Meta & Additive regression & 0.92 & 111.65 & 131.92 & 36.49 & 36.90 \\
4 & Meta & Bagging & 0.91 & 107.83 & 139.80 & 35.24 & 39.11 \\
\hline 5 & Trees & Random forest & 0.95 & 87.08 & 108.45 & 28.46 & 30.34 \\
\hline
\end{tabular}

CC, correlation coefficient; MAE, mean absolute error; RMSE, root mean squared error; RAE, relative absolute error.

Table 4. Prediction for 6 test cases

\begin{tabular}{|c|c|c|c|c|c|c|}
\hline Serial no. & Reinsertion & $\begin{array}{l}\text { Density } \\
\left(\mathrm{kg} / \mathrm{m}^{3}\right)\end{array}$ & $\begin{array}{l}\text { Insertion depth } \\
(\%, \boldsymbol{l})\end{array}$ & $\begin{array}{l}\text { Insertion angle } \\
\qquad\left(^{\circ}, \theta\right)\end{array}$ & $\begin{array}{c}\text { No. of experimental } \\
\text { value }\end{array}$ & $\begin{array}{c}\text { No. of predicted } \\
\text { value }\end{array}$ \\
\hline 1 & 0 & 300 & 100 & 0 & 1,423 & 1,207 \\
\hline 2 & 0 & 80 & 100 & 20 & 226 & 266 \\
\hline 3 & 0 & 160 & 70 & 10 & 370 & 413 \\
\hline 4 & 0 & 240 & 80 & 30 & 610 & 638 \\
\hline 5 & 1 & 300 & 100 & 0 & 1,305 & 1,145 \\
\hline 6 & 1 & 80 & 100 & 20 & 185 & 237 \\
\hline
\end{tabular}



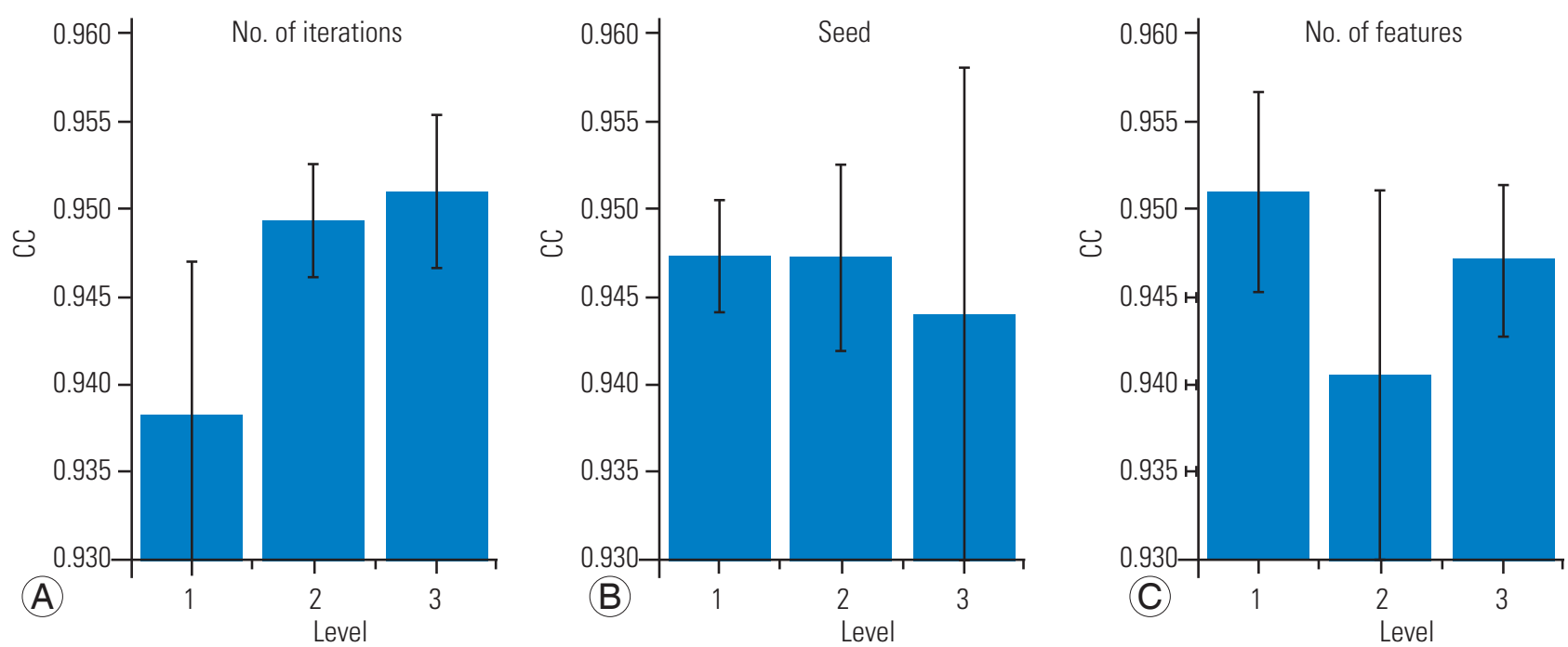

Fig. 2. (A) Variation of CC with the number of iterations. (B) Variation of CC with seed. (C) Variation of CC with the number of features. CC, correlation coefficient.

for the performance of random forest algorithm (0.93). In the lazy class of algorithms, Kstar algorithm (0.89) showed the best performance and in the meta class, it was additive regression (0.92). The experimental tables used for building the model and algorithms were evaluated based on their performance in the error matrix.

\section{Sensitivity analysis of parameters for random forest}

Based on the evaluation of algorithms using different parameters, we found that random forest performed the best, as the values of error matrix were least, and correlation coefficient was the highest. An optimization exercise using the Taguchi Design of Experiments to find the best combination of parameters consisted of three levels of all three factors (Table 3) and a L9 orthogonal array to test the parameters. The levels of all three factors were selected so that they covered the entire range over which significant changes in the value of factors were reflected in the output. The optimization of parameters showed the best combination had a correlation coefficient of 0.96 with seed equal to 6 , iterations equal to 100 and zero features (Fig. 2).

\section{Prediction}

The performance of the model at six test data points evaluated using the combination of parameters for random forest algorithm showed that the algorithm predicted the performance favorably (Table 4). The difference between the experimental and predicted value was $14 \%$, and it was not significant $(p>0.05)$.

\section{Discussion}

The overall objective of the current study was to develop a model to predict pullout strength of pedicle screw for various combinations of density, insertion depth, insertion angle, and reinsertion. The lack of difference in performance between the experimental and predicted value of pullout strength showed that our prediction model was able to satisfy the premise behind its development by machine learning. While analytical models to predict pullout strength based on material properties of the bone and screw thread parameters have been developed by Chapman et al. [20] and Tsai et al. [21], their major limitation is that factors such as insertion angle and reinsertion are not incorporated in the models. A pullout strength calculator that was developed by Varghese et al. [19] and based on a surrogate modeling approach also suffers from limitations since it is a metamodeling approach which cannot be used to develop a machine learning-based decision support system.

One of the applications of our model is to develop a decision support system for pre-surgical planning. The predicted pullout strength value can act as an index for predicting biomechanical stability of the fusion construct. The model can be used to develop tools that enable primary care physicians to identify patients at risk before refereeing to secondary specialists. Further, the model can 
help surgeons to educate their patients regarding availability of various surgical options. Decision tree-based models are used to develop a flowchart for evaluation of surgical outcomes and hence aid in surgical treatment planning. Varghese et al. [22] has proposed a methodology to evaluate the success or failure of pedicle screw fixation based on the predicted value of pullout strength.

The model developed in this study can also assist in understanding the effect of various factors on pullout strength of the pedicle screws. We used only four factors for computing the pullout strength of a pedicle screw using a single screw type. Additional screw thread parameters variables such as thread shape, inner and outer diameter, etc. can be incorporated into the model. Studies have described the effect of thread factors such as pitch, ratio of inner diameter to the outer diameter, and thread type on pullout strength $[5,23,24]$, and thus may be included in our model to predict the pullout strength using similar machine learning approach. Based on all these parameters, an optimization study can also be performed to determine the optimal configuration of screws thread parameters for pedicle screws with higher pullout strengths and novel screw designs $[25,26]$.

Clinically, cement augmentations are carried out on osteoporotic vertebra to increase the strength of bone screw interface $[27,28]$. The model that we developed can also be extended to predicting the outcome of cement augmentation for preoperative decision making, and also to decided whether pedicle screw augmentation is required. Thus, the model developed in this study can help surgeons be better prepared for surgery and the decision would be based on objective parameters rather than subjective ones.

Clinical applications of the model may be envisaged in the near future, wherein algorithms can be trained on a patient database with variables such as age, gender, level of injury, type of screw used, BMD, other physiological parameters, level of activity pre and post-surgery, etc. This model will certainly aid in pre-surgical planning and decision making. Finite element analysis, cadaver experiments and testing on novel surrogate bone models can be incorporated into the database [29]. Additional failure mechanism such as cyclic loading and toggling can be investigated to represent the in vivo condition [30].

The limitation of our study is that only one specific database was used to build the model. In order to widely apply the proposed method, a database consisting of variable datasets and factors is warranted, but currently, there are no available database consisting of pullout strength values of pedicle screws and other variables. Another limitation of the current study is that only pullout failure was considered, and other modes of failures were not investigated. However, we limited the scope of our study to mechanical failures, but additional failure modes can be evaluated based on current proposed methodology.

\section{Conclusions}

A machine learning model using the random forest algorithm constructed to predict the pullout strength of pedicle screws showed a lack of difference in performance between the experimental and predicted values. The model developed in this study can help surgeons be better prepared for surgery and the decision would be based on objective, rather than subjective parameters.

\section{Conflict of Interest}

No potential conflict of interest relevant to this article was reported.

\section{References}

1. Hoy D, Brooks P, Blyth F, Buchbinder R. The epidemiology of low back pain. Best Pract Res Clin Rheumatol 2010;24:769-81.

2. Kurtz SM, Edidin AA. Spine: technology handbook. Amsterdam: Elsevier Academic Press; 2006.

3. Ponnusamy KE, Iyer S, Gupta G, Khanna AJ. Instrumentation of the osteoporotic spine: biomechanical and clinical considerations. Spine J 2011;11:54-63.

4. Varghese V, Venkatesh K, Kumar GS. Pull out strength of pedicle screw in normal and osteoporotic cancellous bone models. Proceedings of the 2014 IEEE Conference on Biomedical Engineering and Sciences (IECBES); 2014 Dec 8-10; Kuala Lumpur, Malaysia. Piscataway (NJ): IEEE; 2014. https://doi. org/10.1109/IECBES.2014.7047541.

5. Varghese V, Kumar GS, Venkatesh K. A finite element analysis based sensitivity studies on pull out strength of pedicle screw in synthetic osteoporotic bone models. Proceedings of the 2016 IEEE-EMBS Conference on Biomedical Engineering and Sciences (IECBES); 2016 Dec 4-7; Kuala Lumpur, Malaysia. Piscataway (NJ): IEEE; 2016. 
6. Varghese V, Saravana Kumar G, Krishnan V. Effect of various factors on pull out strength of pedicle screw in normal and osteoporotic cancellous bone models. Med Eng Phys 2017;40:28-38.

7. Demir T, Basgul C. The pullout performance of pedicle screws. Cham: Springer International Publishing; 2015.

8. Krishnan V, Varghese V, Kumar GS. Comparative analysis of effect of density, insertion angle and reinsertion on pull-out strength of single and two pedicle screw constructs using synthetic bone model. Asian Spine J 2016;10:414-21.

9. Aycan MF, Tolunay T, Demir T, Yaman ME, Usta Y. Pullout performance comparison of novel expandable pedicle screw with expandable poly-ether-etherketone shells and cement-augmented pedicle screws. Proc Inst Mech Eng H 2017;231:169-75.

10. Demir T, Camuscu N, Tureyen K. Design and biomechanical testing of pedicle screw for osteoporotic incidents. Proc Inst Mech Eng H 2012;226:256-62.

11. Yaman O, Demir T, Arslan AK, et al. The comparison of pullout strengths of various pedicle screw designs on synthetic foams and ovine vertebrae. Turk Neurosurg 2015;25:532-8.

12. Kwok AW, Finkelstein JA, Woodside T, Hearn TC, $\mathrm{Hu} \mathrm{RW}$. Insertional torque and pull-out strengths of conical and cylindrical pedicle screws in cadaveric bone. Spine (Phila Pa 1976) 1996;21:2429-34.

13. Reynolds KJ, Cleek TM, Mohtar AA, Hearn TC. Predicting cancellous bone failure during screw insertion. J Biomech 2013;46:1207-10.

14. Mitchell TM. Machine learning. New York (NY): McGraw-Hill; 1997.

15. Winters-Miner LA. Seven ways predictive analytics can improve healthcare [Internet]. Amsterdam: Elsevier; 2014 [cited 2018 Feb 28]. Available from: https://www.elsevier.com/connect/seven-ways-predictive-analytics-can-improve-healthcare.

16. ASTM Subcommittee. ASTM F543-02: standard specification and test methods for metallic medical bone screws. West Conshohocken (PA): ASTM International; 2012. https://doi.org/10.1520/F0543.

17. Hall M, Frank E, Holmes G, Pfahringer B, Reutemann $\mathrm{P}$, Witten IH. The WEKA data mining software: an update. SIGKDD Explor Newsl 2009;11:108. https://doi.org/10.1145/1656274.1656278.

18. Witten IH, Frank E, Trigg LE, Hall MA, Holmes G,
Cunningham SJ. Weka: practical machine learning tools and techniques with Java implementations. Hamilton: University of Waikato; 1999.

19. Varghese V, Ramu P, Krishnan V, Saravana Kumar G. Pull out strength calculator for pedicle screws using a surrogate ensemble approach. Comput Methods Programs Biomed 2016;137:11-22.

20. Chapman JR, Harrington RM, Lee KM, Anderson PA, Tencer AF, Kowalski D. Factors affecting the pullout strength of cancellous bone screws. J Biomech Eng 1996;118:391-8.

21. Tsai WC, Chen PQ, Lu TW, Wu SS, Shih KS, Lin SC. Comparison and prediction of pullout strength of conical and cylindrical pedicle screws within synthetic bone. BMC Musculoskelet Disord 2009;10:44.

22. Varghese V, Krishnan V, Kumar GS. Evaluating pedicle-screw instrumentation using decision-tree analysis based on pullout strength. Asian Spine J 2018;12:611-21.

23. Varghese V, Krishnan V, Saravana Kumar G. Simulation of axial pull out of pedicle screw in synthetic bone models. Proceedings of the 2 nd International Conference on Biomedical Systems, Signals and Images; 2016 Feb; Chennai, India. https://doi. org/10.13140/RG.2.1.3355.8643.

24. Chatzistergos PE, Magnissalis EA, Kourkoulis SK. Numerical simulation of bone screw induced pretension: the cases of under-tapping and conical profile. Med Eng Phys 2014;36:378-86.

25. Demir T, Ormeci MF. New pedicle screw design with expandable shell for low bone quality. J Med Devices 2014;8:020935.

26. Demir T. A new alternative to expandable pedicle screws: expandable poly-ether-ether-ketone shell. Proc Inst Mech Eng H 2015;229:386-94.

27. Chen LH, Tai CL, Lee DM, et al. Pullout strength of pedicle screws with cement augmentation in severe osteoporosis: a comparative study between cannulated screws with cement injection and solid screws with cement pre-filling. BMC Musculoskelet Disord 2011;12:33.

28. Tolunay T, Basgul C, Demir T, Yaman ME, Arslan AK. Pullout performance comparison of pedicle screws based on cement application and design parameters. Proc Inst Mech Eng H 2015;229:786-93.

29. Varghese V, Krishnan V, Saravana Kumar G. Testing pullout strength of pedicle screw using synthetic 
bone models: is a bilayer foam model a better representation of vertebra? Asian Spine J 2018;12:398-406.

30. Aycan MF, Yaman ME, Usta Y, Demir T, Tolunay
T. Investigation of toggling effect on pullout performance of pedicle screws. Proc Inst Mech Eng $\mathrm{H}$ 2018;232:395-402. 